\title{
MASSEY HIGHER PRODUCTS
}

\author{
BY \\ DAVID KRAINES(1)
}

In this paper, we shall investigate some properties of a class of higher order cohomology operations of several variables. These operations, the higher products, were defined by Massey as a generalization of his triple product. There is a correspondence between the higher products and iterated Whitehead products in homotopy groups [5], [11].

It has been noted that the differentials in certain spectral sequences involving the Ext and Tor functors are related to Massey higher products [6], [9]. In particular, the differentials in a spectral sequence relating the cohomology of a space with that of its space of loops are generalized higher products.

In the first part we establish a number of properties of these higher products. These properties indicate the similarities between the higher products and the cup product. In the final section, the higher products are specialized to an operation of one variable. In certain cases, this operation may be evaluated in terms of primary Steenrod operations (Theorems 14, 19). These results are useful in the computation of the higher product structure of a space with coefficients in a field.

1. Definitions. Throughout this paper let $(X, A)$ be a pair of topological spaces and let $R$ be a commutative ring with identity. Also let $u_{1}, \cdots, u_{k}$ be positive dimensional cohomology classes, of dimensions $p_{1}, \cdots, p_{k}$ respectively, in the singular cohomology ring $H^{*}(X, A ; R)$. Finally, let $p(i, j)=\sum_{r=i}^{j}\left(p_{r}-1\right)$.

Under certain conditions, we will be able to define the Massey $k$-fold product $\left\langle u_{1}, \cdots, u_{k}\right\rangle$ as a subset of $H^{p(1, k)+2}(X, A ; R)$. We shall first describe a "higher operation" from a subset of the singular cochains to a subset of a cohomology group.

Let $C^{*}(X, A ; R)$ be the singular cochain complex with the usual associative cup product pairing. Let $a_{1}, \cdots, a_{k}$ be cocycle representatives of $u_{1}, \cdots, u_{k}$ respectively. If $a \in C^{p}(X, A ; R)$, then the symbol $\bar{a}$ will denote $(-1)^{p} a$.

Definition 1. A collection of cochains, $A=(a(i, j))$, for $1 \leqq i \leqq j \leqq k$ and

Received by the editors October 11, 1965.

(1) This research was supported in part by the National Science Foundation grant GP 2497. The author wishes to express his gratitude to Professor E. Spanier for his guidance. 
$(i, j) \neq(1, k)$ is said to be a defining system for the (cochain) product $\left\langle a_{1}, \cdots, a_{k}\right\rangle$ if

$$
\begin{aligned}
& a(i, i)=a_{i} \text { for } i=1, \cdots, k, \\
& a(i, j) \in C^{p(i, j)+1}(X, A ; R)
\end{aligned}
$$

and

$$
\delta a(i, j)=\sum_{r=i}^{j-1} \bar{a}(i, r) a(r+1, j) .
$$

The $p(1, k)+2$ dimensional cocycle, $c(A)$, defined by

$$
c(A)=\sum_{r=1}^{k-1} \bar{a}(1, r) a(r+1, k),
$$

is called the related cocycle of the defining system $A$.

Definition 2. The (cochain) $k$-fold product $\left\langle a_{1}, \cdots, a_{k}\right\rangle$ is said to be defined if there is a defining system for it. If it is defined, then $\left\langle a_{1}, \cdots, a_{k}\right\rangle$ consists of all classes $w \in H^{p(1, k)+2}(X, A ; R)$ for which there exists a defining system $A$ such that $c(A)$ represents $w$.

The above definition of the higher products differs from that given in [4] by the sign $(-1)^{h}$ where $h=\sum_{i=1}^{k-1} i p_{k-i}+(k-2)(k-1) / 2$. This convention reduces the work of checking signs.

THEOREM 3. The operation $\left\langle a_{1}, \cdots, a_{k}\right\rangle$ depends only on the cohomology classes of the cocycles $a_{1}, \cdots, a_{k}$.

Proof. We need only show that $\left\langle a_{1}, \cdots, a_{t}, \cdots, a_{k}\right\rangle=\left\langle a_{1}, \cdots, a_{t}+\delta b, \cdots, a_{k}\right\rangle$ for $t=1, \cdots, k$ and any $b \in C^{p_{t}-1}(X, A ; R)$. Given a defining system $A$ for the former, we shall construct a defining system $A^{\prime}$ for the latter such that $c(A)$ is cohomologous to $c\left(A^{\prime}\right)$. This will imply that

$$
\left\langle a_{1}, \cdots, a_{t}, \cdots, a_{k}\right\rangle \subset\left\langle a_{1}, \cdots, a_{t}+\delta b, \cdots, a_{k}\right\rangle .
$$

Thee reverse inclusion will follow by symmetry.

Define a set of cochains $\left(a^{\prime}(i, j)\right)$ as follows:

$$
\begin{aligned}
& a(t, t)=a_{t}+\delta b, \\
& a^{\prime}(i, t)=a(i, t)+a(i, t-1) b \text { for } i<t, \\
& a^{\prime}(t, j)=a(t, j)-\bar{b} a(t+1, j) \text { for } j>t, \\
& a^{\prime}(i, j)=a(i, j) \text { if } i \neq t \text { and } j \neq t .
\end{aligned}
$$

By a straightforward calculation, it is seen that $A^{\prime}$ is a defining system for $\left\langle a_{1}, \cdots, a_{t}+\delta b, \cdots, a_{k}\right\rangle$. If $1<t<k$, then $c(A)=c\left(A^{\prime}\right)$. If $t=1$, then they differ by $-\delta \bar{b} a(2, k)$. If $t=k$, they differ by $\delta a(1, k-1) b$. Q.E.D. 
The preceding theorem enables us to make the following definition.

Definition 4. A set of cocycles is said to be a defining system for the Massey $k$-fold product $\left\langle u_{1}, \cdots, u_{k}\right\rangle$ if it is one for $\left\langle a_{1}, \cdots, a_{k}\right\rangle$. The Massey $k$-fold product $\left\langle u_{1}, \cdots, u_{k}\right\rangle$ is said to be defined if $\left\langle a_{1}, \cdots, a_{k}\right\rangle$ is defined, in which case $\left\langle u_{1}, \cdots, u_{k}\right\rangle=\left\langle a_{1}, \cdots, a_{k}\right\rangle$ as subsets of $H^{p(1, k)+2}(X, A ; R)$.

The 2-fold product $\left\langle u_{1}, u_{2}\right\rangle$ is the subset consisting of the singular cup product $(-1)^{p_{1}} u_{1} u_{2}$. The 3 -fold product $\left\langle u_{1}, u_{2}, u_{3}\right\rangle$ is defined if and only if the cup products $u_{1} u_{2}=0$ and $u_{2} u_{3}=0$. This is a secondary operation which differs from the Massey triple product by the sign $(-1)^{p_{2}+1}[11]$.

The $k$-fold product is a $(k-1)$ order cohomology operation of $k$ variables. In order for $\left\langle u_{1}, \cdots, u_{k}\right\rangle$ to be defined, it is necessary that the $(k-2)$ order operations $\left\langle u_{1}, \cdots, u_{k-1}\right\rangle$ and $\left\langle u_{2}, \cdots, u_{k}\right\rangle$ be defined and contain the zero element. In general, this condition is not sufficient. There must exist defining systems $\boldsymbol{A}^{\prime}$ and $A^{\prime \prime}$ for $\left\langle u_{1}, \cdots, u_{k-1}\right\rangle$ and $\left\langle u_{2}, \cdots, u_{k}\right\rangle$ respectively, for which not only do the related cocycles of each cobound but also $a^{\prime}(i, j)=a^{\prime \prime}(i, j)$ for $1<i \leqq j<k$. In this case, we say that the $(k-1)$-fold products $\left\langle u_{1}, \cdots, u_{k-1}\right\rangle$ and $\left\langle u_{2}, \cdots, u_{k}\right\rangle$ vanish simultaneously.

The domain of the higher products may be extended somewhat. Let $\bar{X}$ be a topological space and let $\left(X_{i}, A_{i}\right)$ be pairs of subspaces of $\bar{X}$, for $i=1, \cdots, k$, such that $\bigcup_{r=1}^{k} A_{r} \subset \bigcap_{r=1}^{k} X_{r}$. Assume that the triads $\left(\bar{X}, A_{i}, A_{j}\right)$ are proper in the singular cohomology theory for $1 \leqq i, j \leqq k$. This condition will be satisfied if each of the spaces $X_{i}$ and $A_{i}$ are open in $\bar{X}$ or if $\bar{X}$ is a CW complex and each $X_{i}$ and $A_{i}$ are subcomplexes.

Let $u_{1}, \cdots, u_{k}$ be classes in the cohomology groups $H^{p_{1}}\left(X_{1}, A_{1} ; R\right), \cdots, H^{p_{k}}\left(X_{k}, A_{k} ; R\right)$ respectively. As before, under certain conditions it is possible to define the $k$-fold product $\left\langle u_{1}, \cdots, u_{k}\right\rangle$, this time as a subset of $H^{p(1, k)+2}\left(\bigcap_{r=1}^{k} X_{r}, \bigcup_{r=1}^{k} A_{r} ; R\right)$.

2. Properties. The Massey higher products can be viewed as higher order analogues of the cup product. The properties we shall establish below are generalizations of well-known relations satisfied by the cup product.

The first several properties we shall list are functorial in nature. The proofs of these will be immediate from the definitions of the first section.

(2.1) Naturality. Let $(Y, B)$ be a pair of spaces and $R^{\prime}$ a commutative ring with identity. Let $f:(Y, B) \rightarrow(X, A)$ be a map of pairs and $g: R \rightarrow R^{\prime}$ be a map of rings. If $\left\langle u_{1}, \cdots, u_{k}\right\rangle$ is defined, then so is $\left\langle f^{*} g_{\#} u_{1}, \cdots, f^{*} g_{\#} u_{k}\right\rangle$ and $f^{*} g_{\#}\left\langle u_{1}, \cdots, u_{k}\right\rangle$ $c\left\langle f^{*} g_{\#} u_{1}, \cdots, f^{*} g_{\#} u_{k}\right\rangle$. Furthermore if $f^{*}$ and $g_{\#}$ are isomorphisms, then equality holds between the two operations.

(2.2) Other Chain Complexes. The higher products may be defined in an analogous way with the use of any other chain complex, $\bar{C}^{*}(X, A ; R)$, which has an associative product. For example, for certain pairs of spaces, we could have used the simplicial theory, the Čech theory, or the Alexander-Spanier theory instead of the singular theory. 
Assume that there is a chain equivalence between $\bar{C}^{*}(X, A ; R)$ and the singular complex $C^{*}(X, A ; R)$ such that the map from one complex to the other commutes with the cup product. Then the isomorphism induced by the chain equivalence preserves the higher products.

(2.3) Scalar Multiplication. Assume that the $k$-fold product $\left\langle u_{1}, \cdots, u_{k}\right\rangle$ is defined. Then, for any $x \in R$ and $t=1, \cdots, k$, the product $\left\langle u_{1}, \cdots, x u_{t}, \cdots, u_{k}\right\rangle$ is defined and $x\left\langle u_{1}, \cdots, u_{k}\right\rangle \subset\left\langle u_{1}, \cdots, x u_{t}, \cdots, u_{k}\right\rangle$.

(2.4) Loop Suspension. Let $\pi: P X \rightarrow X$ be the path-loop fibration over $X$. Then $E_{A}=\pi^{-1}(A)$ is the space of paths in $X$ starting from the base point and ending in $A$. The relative loop suspension $\sigma: H^{n}(X, A ; R) \rightarrow H^{n-1}\left(E_{A} ; R\right)$ is defined as the composite homomorphism

$$
H^{n}(X, A ; R) \stackrel{\pi^{*}}{\rightarrow} H^{n}\left(P X, E_{A} ; R\right) \stackrel{\delta}{\longleftarrow} H^{n-1}\left(E_{A} ; R\right)
$$

THEOREM 5. Assume that $\left\langle u_{1}, \cdots, u_{k}\right\rangle$ is defined as a subset of $H^{p(1, k)+2}(X, A ; R)$. Then $\sigma\left\langle u_{1}, \cdots, u_{k}\right\rangle$ is the subset of $H^{p(1, k)+1}\left(E_{A} ; R\right)$ consisting solely of the zero element.

Proof. Since $\delta$ is an isomorphism and $\pi^{*}\left\langle u_{1}, \cdots, u_{k}\right\rangle \subset\left\langle\pi^{*} u_{1}, \cdots, \pi^{*} u_{k}\right\rangle$, it suffices to show that the latter higher product consists solely of the zero element. Let $A^{\prime}$ be a defining system for $\left\langle\pi^{*} u_{1}, \cdots, \pi^{*} u_{k}\right\rangle$, and let $j^{\#}: C^{*}\left(P X, E_{4} ; R\right)$ $\rightarrow C^{*}(P X ; R)$ be the canonical injection. Since $j^{\#} a^{\prime}(1,1)$ is a cocycle in the acyclic complex $C^{*}(P X ; R)$, there is a cochain $b_{1}$ of dimension $p_{1}-1$ such that $\delta b_{1}=j^{\#} a(1,1)$. By induction on $j$, there are cochains $b_{j} \in C^{p(1, j)}(P X ; R)$, for $j=1, \cdots, k-1$, such that

$$
\delta b_{j}=j^{\#} a^{\prime}(1, j)+\sum_{r=1}^{j-1} \tilde{b}_{r} j^{\#} a^{\prime}(r+1, j),
$$

since the right hand side can be seen to be a cocycle. A straightforward calculation yields

$$
-\delta \sum_{r=1}^{k-1} \bar{b}_{r} a^{\prime}(r+1, k)=\sum_{r=1}^{k-1}\left(j^{*} \bar{a}^{\prime}(1, r)\right) a^{\prime}(r+1, k)=c(A)^{\prime}
$$

Q.E.D.

The final properties which we shall establish are more algebraic. Their proofs would be straightforward if the cup product were commutative on the cochain level. In general it is not even true that $a b \sim b a$ for singular cochains $a$ and $b$. However there is a pairing of singular cochain groups, the $U_{1}$ product of Steenrod [10], from $C^{p} \otimes C^{q}$ to $C^{p+q-1}$ such that 


$$
\delta\left(a \cup_{1} b\right)=-\overline{a b}+(-1)^{p q} \overline{b a}+\delta a \cup_{1} b+\bar{a} \cup_{1} \delta b
$$

where $a \in C^{p}$ and $b \in C^{q}$. Furthermore if $c \in C^{r}$ then there is a formula of $\mathrm{G}$. Hirsch [3] which states

$$
(a b)] \cup \cup_{1} c=a\left(b \cup_{1} c\right)+(-1)^{q(r-1)}\left(a \cup_{1} c\right) b .
$$

TheOREM 6. Assume $\left\langle u_{1}, \cdots, u_{k}\right\rangle$ is defined in $H^{p(1, k)+2}(X, A ; R)$ and let $v \in H^{q}(X, A ; R)$. Then the $k$-fold product $\left\langle u_{1}, \cdots, u_{t} v, \cdots, u_{k}\right\rangle$ is defined, for $t=1, \cdots, k$, as a subset of $H^{q+p(1, k)+2}(X, A ; R)$. Furthermore the following relations are satisfied.

$$
\begin{aligned}
\left\langle u_{1}, \cdots, u_{k}\right\rangle v & \subset\left\langle u_{1}, \cdots, u_{k} v\right\rangle, \\
v\left\langle u_{1}, \cdots, u_{k}\right\rangle & \subset(-1)^{q}\left\langle v u_{1}, \cdots, u_{k}\right\rangle, \\
\left\langle u_{1}, \cdots, u_{t} v, \cdots, u_{k}\right\rangle & \cap(-1)^{q}\left\langle u_{1}, \cdots, v u_{t+1}, \cdots, u_{k}\right\rangle \neq \varnothing .
\end{aligned}
$$

These relations may be interpreted as equalities modulo the sum of the indeterminacies.

Proof. Let $A=(a(i, j))$ be a defining system for $\left\langle u_{1}, \cdots, u_{k}\right\rangle$ and let $b$ be a cocycle representative of $v$. Set

$$
a_{1}(i, j)= \begin{cases}a(i, j) & \text { if } j<k, \\ a(i, k) b & \text { if } j=k .\end{cases}
$$

Clearly $A_{1}$ is a defining system for $\left\langle u_{1}, \cdots, u_{k} v\right\rangle$ and $c(A) b=c\left(A_{1}\right)$. This proves (2.7). To prove $(2.8)$, set

$$
a_{2}(i, j)= \begin{cases}a(i, j), & i>1, \\ b a(1, j), & i=1\end{cases}
$$

and note that $\bar{b} c(A)=c\left(A_{2}\right)$.

The proof of relation (2.9) requires the use of the $U_{1}$ product. Set

$$
a_{3}(i, j)= \begin{cases}a(i, t) b & \text { if } j=t, \\ a(i, j) & \text { if } i>t \text { or } j<t, \\ (-1)^{m(j)}\left[a(i, j) b+\sum_{r=t}^{j-1} a(i, r)(\bar{a}(r+1, j) \cup(\bar{b})] \text { for } i \leqq t<j,\right.\end{cases}
$$

where $m(j)=q p(t+1, j)$. To verify that $A_{3}$ is a defining system for $\left\langle u_{1}, \cdots, u_{t}, \cdots, u_{k}\right\rangle$, we need only compute $\delta a_{3}(i, j)$ for $i \leqq t<j$. Using (2.5) and (2.6) 


$$
\begin{aligned}
\delta a_{3}(i, j)= & (-1)^{m(j)} \sum_{r=i}^{j-1} \tilde{a}(i, r) a(r+1, j) b \\
& +\sum_{r=t}^{j-1} \sum_{s=i}^{r-1} \tilde{a}(i, s) a(s+1, r)\left(\bar{a}(r+1, j) \cup_{1} \bar{b}\right) \\
& +\sum_{r=t}^{j-1} \bar{a}(i, r)\left(-a(r+1, j) b+(-1)^{q(p(r+1, j)+1)} b a(r+1, j)\right) \\
& \left.-\sum_{r=t}^{j-1} \sum_{s=r+1}^{j-1} \bar{a}(i, r)\left((a(r+1, s) \bar{a}(s+1, j)) \cup_{1} \bar{b}\right)\right] \\
= & (-1)^{m(j)} \sum_{s=i}^{t-1} \tilde{a}(i, s)\left[a(s+1, j) b+\sum_{r=t}^{j-1} a(s+1, r)(\tilde{a}(r+1, j) \cup 1, \bar{b})\right] \\
& +\sum_{s=t}^{j-1}(-1)^{m(s)}\left[\tilde{a}(i, s) \bar{b}-\sum_{r=t}^{s-1} \tilde{a}(i, r)\left(a(r+1, s) \cup_{1} b\right)\right] a(s+1, j) \\
= & \sum_{r=i}^{j-1} \bar{a}_{3}(i, r) a_{3}(r+1, j) .
\end{aligned}
$$

Finally set

$$
a_{4}(i, j)=\left\{\begin{array}{l}
a_{3}(i, j) \quad \text { if } i \neq t+1 \text { and } j \neq t \\
a(i, t) \quad \text { if } j=t \\
\bar{b} a(t+1, j) \quad \text { if } i=t+1
\end{array}\right.
$$

Then $A_{4}$ is a defining system for $(-1)^{q}\left\langle u_{1}, \cdots, v u_{t+1}, \cdots, u_{k}\right\rangle$ and $c\left(A_{3}\right)=c\left(A_{4}\right)$.

Q.E.D.

COROLlARY 7. If $\left\langle u_{1}, \cdots, u_{k}\right\rangle$ is defined and $v_{1}, \cdots, v_{k}$ are arbitrary cohomology classes, then $\left\langle u_{1} v_{1}, \cdots, u_{k} v_{k}\right\rangle$ is defined. Furthermore if the latter higher product consists of a single class for any choice of $v_{1}, \cdots, v_{k}$ (the operation has no indeterminancy), then

$$
\left\langle u_{1} v_{1}, \cdots, u_{k} v_{k}\right\rangle= \pm\left\langle u_{1}, \cdots, u_{k}\right\rangle v_{1} \cdots v_{k} .
$$

Finally we prove two analogues of the commutativity properties of the cup product.

THEOREM 8. Assume $\left\langle u_{1}, \cdots, u_{k}\right\rangle$ is defined. Then $\left\langle u_{k}, \cdots, u_{1}\right\rangle$ is defined and

$$
\left\langle u_{1}, \cdots, u_{k}\right\rangle=(-1)^{h}\left\langle u_{k}, \cdots, u_{1}\right\rangle \text {, }
$$

where

$$
h=\sum_{1 \leqq r<s \leqq k} p_{r} p+(k-1) \sum_{r=1}^{k} p_{r}+\frac{(k-1)(k-2)}{2} .
$$


Proof. In Lemma 9 at the end of this proof we shall show by means of acyclic model theory that there exists functorial homomorphisms $E_{n}$ from the $n$-fold tensor product $\left(C^{*}(X, A ; R)\right)^{n}$ to $C^{*}(X, A ; R)$ of homogeneous degree $1-n$, for $n=1,2, \cdots$, such that

(a) $E_{1}$ is the identity,

(b) $\delta E_{n}+(-1)^{n} E_{n} \delta=G_{n}$ with

(c)

$$
\begin{aligned}
G_{n}\left(b_{1} \otimes \cdots \otimes b_{n}\right)= & \sum_{s=1}^{n-1}(-1)^{s} E_{n-1}\left(b_{1} \otimes \cdots \otimes b_{s} b_{s+1} \otimes \cdots \otimes b_{n}\right) \\
& +\sum_{r=1}^{n-1}(-1)^{m(r)} E_{n-r}\left(b_{r+1} \otimes \cdots \otimes b_{n}\right) E_{r}\left(b_{1} \otimes \cdots \otimes b_{r}\right)
\end{aligned}
$$

where $m(r)=\left(q_{r+1}+\cdots+q_{n}\right)\left(q_{1}+\cdots+q_{r}-r+1\right)+n(r+1)$ and $q_{i}=\operatorname{dim} b_{i}$.

Let $A$ be a defining system for $\left\langle u_{1}, \cdots, u_{k}\right\rangle$. Define a system of cochains

$$
a^{\prime}(i, j)=(-1)^{h(i, j)} \sum_{n=1}^{j-i+1} \sum_{\alpha \in I(n)} E_{n}\left(\alpha_{1} \otimes \cdots \otimes \alpha_{n}\right)
$$

where $I(n)$ is the set of all sequences

$$
\begin{aligned}
\alpha & =\left\{i=m_{1}<m_{2}<\cdots<m_{n+1}=j+1\right\}, \\
\alpha_{s} & = \begin{cases}a\left(m_{s}, m_{s+1}-1\right) & \text { if } n-s \text { is even, } \\
\bar{a}\left(m_{s}, m_{s+1}-1\right) & \text { if } n-s \text { is odd, }\end{cases}
\end{aligned}
$$

and

$$
h(i, j)=\sum_{i \leqq s<t \leqq j} p_{s} p_{t}+(j-i) \sum_{s=i}^{j} p_{s}+\frac{(j-i)(j-i-1)}{2} .
$$

Clearly $a^{\prime}(i, i)=a_{i}$. We shall verify that

$$
\begin{aligned}
(-1)^{h(i, j)} \delta a^{\prime}(i, j)= & \sum_{n=1}^{j-i+1} \sum_{\alpha \in I(n)}\left[\sum_{r=1}^{n}(-1)^{n+1} E_{n}\left(\bar{\alpha}_{1} \otimes \cdots \otimes \delta \alpha_{r} \otimes \cdots \otimes \alpha_{n}\right)\right. \\
& +\sum_{s=1}^{n-1}(-1)^{s} E_{n-1}\left(\alpha_{1} \otimes \cdots \otimes \alpha_{s} \alpha_{s+1} \otimes \cdots \otimes \alpha_{n}\right) \\
& \left.+\sum_{t=1}^{n-1}(-1)^{m(t, a)} E_{n-t}\left(\alpha_{t+1} \otimes \cdots \otimes \alpha_{n}\right) E_{t}\left(\alpha_{1} \otimes \cdots \otimes \alpha_{t}\right)\right] .
\end{aligned}
$$

The first two summations cancel each other. The last can be seen to be the right hand side of equation (2.10) after checking signs. Thus $A^{\prime}$ is a defining system for $\left\langle u_{k}, \cdots, u_{1}\right\rangle$. 
Moreover, a similar computation yields

$$
\delta \sum_{n=2}^{k} \sum_{\alpha \in I(n)} E_{n}\left(\alpha_{1} \otimes \cdots \otimes \alpha_{n}\right)+c(A)=(-1)^{h} c\left(A^{\prime}\right) .
$$

This proves that $\left\langle u_{1}, \cdots, u_{k}\right\rangle \subset(-1)^{h}\left\langle u_{k}, \cdots, u_{1}\right\rangle$. The reverse inclusion follows by symmetry.

To complete the proof, we need the following lemma. Let $C^{*}$ be the singular cochain complex of some pair of spaces. Let $D:\left(C^{*}\right)^{2} \rightarrow C^{*}$ represent a cup product pairing (induced by some diagonal approximation $\Delta: C_{*} \rightarrow\left(C_{*}\right)^{2}$ ) and let $T:\left(C^{*}\right)^{2} \rightarrow\left(C^{*}\right)^{2}$ be the cochain automorphism defined by $T(a \otimes b)=(-1)^{p q} b \otimes a$, where $\operatorname{dim} a=p$ and $\operatorname{dim} b=q$.

LEMMA 9. For each $n=1,2, \cdots$, there is a functorial homomorphism $E_{n}:\left(C^{*}\right)^{n} \rightarrow C^{*}$ of homogeneous degree $1-n$, satisfying the conditions

(a) $E_{1}: C^{*} \rightarrow C^{*}$ is the identity,

(b) $\delta E_{2}+E_{2} \delta=D(1-T)=G_{2}$,

(c) $\delta E_{n}+(-1)^{n} E_{n} \delta=G_{n}$ for $n>2$,

where, for $n>2, G_{n}$ is any functorial homomorphism of homogeneous degree $2-n$ with the following properties: $G_{n}$ is defined whenever $E_{1}, \cdots, E_{n-1}$ are; $\delta G_{n}=(-1)^{n} G_{n} \delta$; and if $E_{k}$ maps $(k-1)$-cochains of $\left(C^{*}\right)^{k}$ to 0 in $C^{0}$ for all $k=2, \cdots, n-1$, then $G_{n}$ maps $(n-2)$-cochains of $\left(C^{*}\right)^{n}$ to 0 in $C^{0}$.

Proof. We shall prove the result by constructing chain maps $E^{n}: C_{*} \rightarrow\left(C_{*}\right)^{n}$ which will be dual to $E_{n}$. Let $\Delta: C_{*} \rightarrow\left(C_{*}\right)^{2}$ be the diagonal approximation which induces $D$ and let $T^{\prime}$ be the dual to $T$. Then $\Delta-T^{\prime} \Delta$ is a functorial chain map from $C_{*}$ to $\left(C_{*}\right)^{2}$ which sends 0 -chains to 0 . The zero homomorphism from $C_{*}$ to $\left(C_{*}\right)^{2}$ is a functorial chain map which agrees with $\Delta-T^{\prime} \Delta$ on the zero chains. By the acyclic model theorem [7, Chapter 4], the two maps are functorially chain homotopic. Thus there is a map $E^{2}: C_{*} \rightarrow\left(C_{*}\right)^{2}$ such that $\partial E^{2}+E^{2} \partial=\Delta-T \Delta$. We may choose $E^{2}$ so that it sends 0 -chains to 0 .

Now assume that $E^{k}: C_{*} \rightarrow\left(C_{*}\right)^{k}$ is defined, of homogeneous degree $k-1$ for all $k<n$, such that

(a') $E^{k}$ is functorial,

(b') $E^{k}$ sends 0 -chains to 0 , and

(c') $(-1)^{k} \partial E^{k}+E^{k} \partial=G^{k}$, where $G^{k}$ is the dual of $G_{k}$.

By hypothesis, $G^{n}$ is defined, functorial and sends zero dimensional chains to 0 in the $(n-2)$-chain group of $\left(C_{*}\right)^{n}$. The map $G^{n}$ is either a chain map (if $n$ is even), or can be made into one by a sign trick. By the acyclic model theorem, $G^{n}$ is chain homotopic to the zero map. That is, there is a functorial map $E^{n}$ which satisfies $\left(a^{\prime}\right),\left(b^{\prime}\right)$, and $\left(c^{\prime}\right)$. The dual map to $E^{n}$ clearly satisfies (a), (b), and (c).

Q.E.D.

THEOREM 10. Assume that the $k$-fold products $\left\langle u_{t}, \cdots, u_{k}, u_{1}, \cdots, u_{t-1}\right\rangle$, for 
$t:=1, \cdots, k$, are simultaneously defined. Then there are classes $x_{t} \in\left\langle u_{t}, \cdots, u_{t-1}\right\rangle$, for $t=1, \cdots, k$, such that

$$
\begin{gathered}
\sum_{t=1}^{k}(-1)^{\pi(t)} x_{t}=0 \text { where } \\
\pi(1)=\sum_{r=1}^{k}(k-r) p_{r}+(k-1)
\end{gathered}
$$

and

$$
\begin{array}{r}
\pi(t)=\left(p_{1}+\cdots+p_{t-1}+k\right)\left(p_{t}+\cdots+p_{k}\right)+\sum_{r=1}^{k}(t-1-r) p_{r}+t(k-1) \\
\text { for } t>1 .
\end{array}
$$

Proof. The condition that the higher products be defined simultaneously implies that there exists cochains $(a(i, j))$ for $1 \leqq i, j \leqq k,(i, j) \neq(1, k)$ and $(i, j) \neq(t, t-1)$ such that $a(i, i)$ is a cocycle representative of $u_{i}$ and

$$
\delta a(i, j)=\Sigma \bar{a}(i, r) a(r+1, j)
$$

where, if $i<j$, then the summation is taken over all $r$ with $i \leqq r<j$, and if $i>j$, then the summation is taken over all $r$ with $i \leqq r$ or $r<j$ and $a(k+1, j)$ is interpreted as $a(1, j)$. If $A_{t}$ is the subset of these cochains which is a defining system for $\left\langle u_{t}, \cdots, u_{t-1}\right\rangle$ then $c\left(A_{t}\right)=\sum_{r \neq t-1} \bar{a}(t, r) a(r+1, t-1)$.

By Lemma 9, there is a set of functorial "permuting" homomorphisms $E_{n}^{\prime}:\left(C^{*}\right)^{n} \rightarrow C^{*}$ of homogeneous degree $1-n$ satisfying the following conditions:

(a) $E_{1}$ is the identity,

(b) $\delta E_{n}^{\prime}+(-1)^{n} E_{n}^{\prime} \delta=G_{n}^{\prime}$ where

(c)

$$
\begin{aligned}
G_{n}\left(b_{1} \otimes \cdots \otimes b_{n}\right)= & (-1)^{m} E_{n-1}^{\prime}\left(b_{2} \otimes \cdots \otimes b_{n} b_{1}\right) \\
& +\sum_{s=1}^{n-1}(-1)^{s} E_{n-1}^{\prime}\left(b_{1} \otimes \cdots \otimes b_{s} b_{s+1} \otimes \cdots \otimes b_{n}\right)
\end{aligned}
$$

where $m=q_{1}\left(q_{2}+\cdots+q_{n}\right)$ and $q_{r}=\operatorname{dim} b_{r}$. Consider the cochain

$$
c=\sum_{n=2}^{k} \sum_{\beta \in J(n)}(-1)^{\pi(\beta)} E_{n}^{\prime}\left(\beta_{1} \otimes \cdots \otimes \beta_{n}\right)
$$

where $J(n)$ is the set of all sequences

$$
\begin{aligned}
& \beta=\left\{1 \leqq m_{1}<m_{2}<\cdots<m_{n} \leqq k\right\} ; \\
& \beta_{n}= \begin{cases}a\left(m_{n}, m_{1}-1\right), & m_{1}>1, \\
a\left(m_{n}, k\right), & m_{1}=1 ;\end{cases} \\
& \beta_{s}= \begin{cases}a\left(m_{s}, m_{s+1}-1\right) & \text { if } n-s \text { is even, } s<n, \\
\bar{a}\left(m_{s}, m_{s+1}-1\right) & \text { if } n-s \text { is odd, } s<n \text { and } \pi(\beta)=\pi\left(m_{1}\right) .\end{cases}
\end{aligned}
$$




$$
\begin{aligned}
& \delta c=\sum_{n=2}^{k} \sum_{\beta \in J(n)}(-1)^{\pi(\beta)}\left[\sum_{r=1}^{n}(-1)^{n+1} E_{n}^{\prime}\left(\bar{\beta}_{1} \otimes \cdots \otimes \delta \beta_{r} \otimes \cdots \otimes \beta_{n}\right)\right. \\
&+(-1)^{m(\beta)} E_{n-1}^{\prime}\left(\beta_{2} \otimes \cdots \otimes \beta_{n} \beta_{1}\right) \\
&\left.+\sum_{s=1}^{n-1}(-1)^{s} E_{n-1}^{\prime}\left(\beta_{1} \otimes \cdots \otimes \beta_{s} \beta_{s+1} \otimes \cdots \otimes \beta_{n}\right)\right] \\
&= \sum_{\beta \in J(2)}(-1)^{\pi(\beta)+m(\beta)} E_{1}\left(\beta_{2} \beta_{1}\right)-(-1)^{\pi(\beta)} E_{1}\left(\beta_{1} \beta_{2}\right) \\
&=-\sum_{t=1}^{k} \sum_{r \neq t-1}(-1)^{\pi(t)} \bar{a}(t, r) a(r+1, t-1) \\
&=-\sum_{t=1}^{k}(-1)^{\pi(t)} c\left(A_{t}\right) .
\end{aligned}
$$

3. The operation $\langle u\rangle^{k}$. Let $u \in H^{m}(X, A ; R)$ be a class such that $u^{2}=0$. Let $a$ be a cocycle representative of $u$. Then the triple product $\langle u, u, u\rangle=\langle a, a, a\rangle$ is defined as a coset of $u H^{2 m-1}(X, A ; R)$ in $H^{3 m-1}(X, A ; R)$.

We may, however, restrict the notion of a defining system for this particular case by requiring that $a(1,2)=a(2,3)$. In other words, choose a cochain $a(2)$ so that $\delta a(2)=\bar{a} a$. Then $\bar{a}(2) a+\bar{a} a(2)$ is a cocycle representative of $\langle a, a, a\rangle$. Any other choice of $a(2)$ would differ by a cocycle $b \in C^{2 m-1}(X, A ; R)$. Thus $(\bar{a}(2)+\bar{b}) a+\bar{a}(a(2)+b)$ also represents $\langle a, a, a\rangle$ in this restricted sense. Since $b$ is an odd dimensional cocycle, the two representatives of $\langle a, a, a\rangle$ differ by the coboundary $-b a+a \bar{b}$. Thus, with this restricted definition, the triple product has no indeterminacy. We shall call this restricted operation $\langle a\rangle^{3}$. Clearly $\langle a\rangle^{3} \subset\langle a, a, a\rangle$.

Definition 11. A system of cochains $A^{*}=(a(n)), n=1, \cdots, k-1$, satisfying

$$
\begin{aligned}
a(n) & \in C^{n(m-1)+1}(X, A ; R), \\
a(1) & =a \text { is a cocycle, } \\
\delta a(n) & =\sum_{r=1}^{n-1} \bar{a}(r) a(n-r),
\end{aligned}
$$

is called a defining system for $\langle a\rangle^{k}$. The cocycle

$$
c\left(A^{*}\right)=\sum_{r=1}^{k-1} \tilde{a}(r) a(k-r)
$$

is called its related cocycle. Then $\langle a\rangle^{k}$ is said to be defined if there is a defining 
system for it, in which case $\langle a\rangle^{k}$ consists of all classes $w \in H^{k(m-1)+2}(X, A ; R)$ for which there is a defining system $A^{*}$ such that $c\left(A^{*}\right)$ represents $w$.

THEOREM 12. The operation $\langle a\rangle^{k}$ depends only on the cohomology class of $a$.

Proof. Let $A^{*}$ be a defining system for $\langle a\rangle^{k}$ and let $b$ be an $(m-1)$-cochain. We shall construct a defining system $A^{\prime *}$ for $\langle a+\delta b\rangle^{k}$ such that the related cocycle of $A^{*}$ is cohomologous to that of $A^{\prime *}$. Define $a^{\prime}(n)$ inductively by

$$
\begin{aligned}
& a^{\prime}(1)=a+\delta b, \\
& a^{\prime}(n)=a(n)-\bar{b} a^{\prime}(n-1)+a(n-1) b, n \geqq 2 .
\end{aligned}
$$

Clearly $A^{\prime *}$ satisfies (3.1) and (3.2). By induction on $n$ we shall show that $A^{\prime *}$ satisfies (3.3). This is immediate if $n=2$. Assume true for $n-1$. Then, on the one hand

$$
\begin{aligned}
\delta a^{\prime}(n)= & \sum_{r=1}^{n-1} \tilde{a}(r) a(n-r)-(\delta \bar{b}) a^{\prime}(n-1) \\
& -\sum_{r=2}^{n-1} b \bar{a}^{\prime}(r-1) a^{\prime}(n-r)+\sum_{r=2}^{n-1} \tilde{a}(r-1) a(n-r) b+\bar{a}(n-1) \delta b .
\end{aligned}
$$

On the other hand

$$
\begin{aligned}
& \sum_{r=1}^{n-1} \bar{a}^{\prime}(r) a^{\prime}(n-r)=\sum_{r=1}^{n-1} \bar{a}(r) a(n-r)+\bar{\delta} \bar{b} a^{\prime}(n-1) \\
& \quad+\sum_{r=2}^{n-1}\left[-b \bar{a}^{\prime}(r-1)+\bar{a}(r-1) \bar{b}\right] a^{\prime}(n-r) \\
& \quad+\sum_{r=1}^{n-2} \bar{a}(r)\left[-\bar{b} a^{\prime}(n-r-1)+a(n-1-r) b\right]+\bar{a}(n-1) \delta b .
\end{aligned}
$$

By reindexing where necessary, the two equations are seen to be equal. A similar calculation shows

$$
\sum_{r=1}^{k-1} \tilde{a}^{\prime}(r) a^{\prime}(k-r)=\sum_{r=1}^{k-1} \bar{a}(r) a(k-r)+\delta\left[-\bar{b} a^{\prime}(k-1)+a(k-1) b\right],
$$

and so the related cocycles are cohomologous. Q.E.D.

Definition 13. Let $a$ be a cocycle representative of $u$. A set of cocycles is said to be a defining system for $\langle u\rangle^{k}$ if it is one for $\langle a\rangle^{k}$. If $\langle a\rangle^{k}$ is defined, then so is $\langle u\rangle^{k}$ and $\langle u\rangle^{k}=\langle a\rangle^{k}$ as subsets of $H^{k(m-1)+2}(X, A ; R)$.

We establish some results concerning these operations $\langle u\rangle^{k}$. Let $p$ be an odd prime and let $\beta$ be the Bockstein operator associated with the exact coefficient sequence $0 \rightarrow Z_{p} \rightarrow Z_{p^{2}} \rightarrow Z_{p} \rightarrow 0$. Furthermore, let $P^{m}$ be the Steenrod $p$ th power $P^{m}: H^{q}\left(X ; Z_{p}\right) \rightarrow H^{q+2 m(p-1)}\left(X ; Z_{p}\right)$. 
THEOREM 14. If $u \in H^{2 m+1}\left(X ; Z_{p}\right)$, then $\langle u\rangle^{p}$ is defined as a single class in $H^{2 m p+2}\left(X ; Z_{p}\right)$ and $\langle u\rangle^{p}=-\beta P^{m} u$.

We first prove some theorems leading up to this result. Note that if $\langle u\rangle^{k}$ is defined, then the $k$-fold product $\langle u, \cdots, u\rangle$ is defined and $\langle u\rangle^{k} \subset\langle u, \cdots, u\rangle$. Also $\langle u\rangle^{k}$ is defined if and only if $\langle u\rangle^{k-1}$ is defined and contains the zero element.

THEOREM 15. Let $Q$ be the rationals. Then, if $u \in H^{2 m+1}(X ; Q),\langle u\rangle^{k}$ is defined, and vanishes in $H^{2 m k+2}(X ; Q)$ for each $k=2,3, \cdots$. Let $p$ be an odd prime. Then if $u \in H^{2 m+1}\left(X ; Z_{p}\right),\langle u\rangle^{k}$ is defined and vanishes in $H^{2 m k+2}\left(X ; Z_{p}\right)$ for each $k=2,3, \cdots, p-1$, and therefore $\langle u\rangle^{p}$ is defined.

Proof. Let $a$ be a cocycle representative of $u$ in $C^{2 m+1}(X ; R)$, where $R$ is $Q$ or $Z_{p}$. By means of the Steenrod $\cup_{1}$-product $(2.5)$ we shall explicitly construct a defining system $(a(n))$ for $\langle u\rangle^{k}$.

If $R=Q$ and $n$ is arbitrary, or if $R=Z_{p}$ and $n<p$, define by induction on $n$, $a(1)=a$, and $a(n)=(1 / n)\left[a(n-1) \cup_{1} a\right]$. It suffices to verify that $\delta a(n)$ $=\sum_{r=1}^{n-1}-a(r) a(n-r)$. Note $\operatorname{dim} a(r)$ is odd for all $r$.

By induction on $n$,

$$
\begin{aligned}
\delta a(n) & =(1 / n) \delta\left(a(n-1) \cup_{1} a\right) \\
& =(1 / n)\left[-a(n-1) a a(n-1)-\sum_{r=1}^{n-2}(a(r) a(n-1-r)) \cup_{1} a\right] .
\end{aligned}
$$

By the formula of G. Hirsch (2.6),

$$
\begin{aligned}
\sum_{r=1}^{n-2}(a(r) a(n-1-r)) \cup_{1} a \\
\quad=\sum_{r=1}^{n-2}\left[a(r)\left(a(n-1-r) \cup_{1} a\right)+\left(a(r) \cup_{1} a\right) a(n-1-r)\right] \\
\quad=\sum_{r=1}^{n-2}[a(r)(n-r) a(n-r)+(r+1) a(r+1) a(n-1-r)] \\
\quad=\sum_{r=2}^{n-2}[n a(r) a(n-r)]+[(n-1)(a(n-1) a(1)+a(1) a(n-1))] .
\end{aligned}
$$

Substituting this result into the above, we see that $(a(n))$ is a defining system for $\langle u\rangle^{k}$. Q.E.D.

We now show that $\langle u\rangle^{p}$ is defined without indeterminacy.

LEMMA 16. Let $u \in H^{2 m+1}\left(X ; Z_{p}\right)$ and assume $\langle u\rangle^{k}$ is defined. Let $A^{*}$ be a defining system for it. If $b$ is a cocycle of dimension $2 m s+1$, where ps $>k$, then there exists a defining system $A^{\prime *}$ for $\langle u\rangle^{k}$ such that $a^{\prime}(n)=a(n)$ if $n<s$, $a^{\prime}(s)=a(s)+b$, and $c\left(A^{*}\right) \sim c\left(A^{\prime *}\right)$. 
Proof. For each pair of integers $(t, q), t<k$, define odd dimensional cochains $c_{t, q}$ by induction on $q$ as follows:

$$
\begin{aligned}
& c_{t, q}=0 \text { if } t<0 \text { or } q<0, \text { or } t=q=0, \\
& c_{t, 0}=a(t) \text { if } t \geqq 1, \\
& c_{0,1}=b, \\
& c_{t, q}=\left(c_{t, q-1} \cup_{1} b\right) \text { otherwise. }
\end{aligned}
$$

Now we may define cochains

$$
a^{\prime}(n)=\sum_{q=0}^{[n / s]} \frac{1}{q !} c_{n-q s, q} \text { for } n=1, \cdots, k-1,
$$

since $[n / s]<p$. If $n<s$, then $a^{\prime}(n)=a(n)$. Also, $a^{\prime}(s)=a(s)+b$. To verify (3.3) we need the following formula

$$
\delta c_{t, q}=-\sum_{r=0}^{q} \sum_{j=0}^{t}\left(\begin{array}{l}
q \\
r
\end{array}\right) c_{j, r} c_{t-j, q-r},
$$

which we establish by induction on $q$. For $q=0$, the formula reduces to

$$
\delta a(t)=-\sum_{j=1}^{t-1} a(j) a(t-j)
$$

Assume now the formula holds for $q$, then

$$
\begin{aligned}
\delta c_{t, q+1} & =\delta\left(c_{t, q} \cup_{1} b\right) \\
& =-c_{t, q} b-b c_{t, q}-\sum_{r=0}^{q} \sum_{j=0}^{t}\left(\begin{array}{l}
q \\
r
\end{array}\right)\left(c_{j, r} c_{t-j, q-r}\right) \cup_{1} b \\
& =-c_{t, q} b-b c_{t, q}-\sum_{r=0}^{q} \sum_{j=0}^{t}\left(\begin{array}{l}
q \\
r
\end{array}\right)\left[c_{j, r}\left(c_{t-j, q-r} \cup_{1} b\right)+\left(c_{j, r} \cup_{1} b\right) c_{t-j, q-r}\right] .
\end{aligned}
$$

(If $(j, r) \neq(0,0)$, then $c_{j, r} \cup_{1} b=c_{j, r+1}$ and $c_{0,0}=0$ )

$$
\begin{aligned}
& =-\sum_{r=0}^{q} \sum_{j=0}^{t}\left(\begin{array}{c}
q \\
r
\end{array}\right)\left[c_{j, r} c_{t-j, q-r+1}+c_{j, r+1} c_{t-j, q-r}\right] \\
& =-\sum_{r=0}^{q+1} \sum_{j=0}^{t}\left[\left(\begin{array}{c}
q+1 \\
r
\end{array}\right) c_{j, r} c_{t-j, q-r+1}\right] .
\end{aligned}
$$

This completes the induction step for the formula. 
Now for all $n, 1 \leqq n \leqq k$,

$$
\begin{aligned}
\sum_{i=1}^{n-1} a^{\prime}(i) a^{\prime}(n-i) & =\sum_{i=1}^{n-1} \sum_{r=0}^{[i / s]} \frac{1}{r !} c_{i-r s, r} \sum_{\rho=0}^{[(n-i) / s]} \frac{1}{\rho !} c_{n-i-\rho s, \rho} \\
& =\sum_{i=1}^{n-1} \sum_{r=0}^{[i / s]} \sum_{\rho=0}^{[(n-i) / s]} \frac{1}{r ! \rho !} c_{i-r s, r} c_{n-i-\rho s, \rho}
\end{aligned}
$$

(Reverse the order of the outer two sums)

$$
=\sum_{r=0}^{[n / s]} \sum_{i=r s}^{n-1} \sum_{\rho=0}^{[(n-i) / s]} \frac{1}{r ! \rho !} c_{i-r s, r} c_{n-i-\rho s, \rho} .
$$

(Reverse the inner two sums and set $j=i-r s$ )

$$
=\sum_{r=0}^{[n / s]} \sum_{\rho=0}^{[n / s]-r} \sum_{j=0}^{n-(r+\rho) s} \frac{1}{r ! \rho !} c_{j, r} c_{n-(r+\rho) s-j, \rho}
$$

(Set $q=r+\rho$ and sum over $q$ )

$$
=\sum_{q=0}^{[n / s]} \sum_{r=0}^{q} \sum_{j=0}^{n-q s} \frac{1}{r !(q-r) !} c_{j, r} c_{n-q s-j, q-r}
$$

$\left(\right.$ Since $\left(\begin{array}{l}q \\ r\end{array}\right)=\frac{q !}{r !(q-r) !}$, and by formula 3.5$)$

$$
=-\sum_{j=1}^{n-1} a(j) a(n-j)-\sum_{q=1}^{[n / s]} \frac{1}{q !} \delta c_{n-q s, q} .
$$

If $n<k$, then the last line is equal to $\delta a^{\prime}(n)$. This proves simultaneously that $A^{\prime *}$ is a defining system for $\langle u\rangle^{k}$ and that the related cocycles of $A^{*}$ and $A^{\prime *}$ are cohomologous. Q.E.D.

THEOREM 17. For an odd prime $p$, let $u \in H^{2 m+1}\left(X ; Z_{p}\right)$. Then $\langle u\rangle^{p}$ is defined as a single class of $H^{2 m p+2}\left(X ; Z_{p}\right)$.

Proof. Let $A^{*}$ and $A^{\prime *}$ be two defining systems for $\langle a\rangle^{p}$, where $a$ is a cocycle representative of $u$. We shall show that their related cocycles are cohomologous.

For this, it suffices to construct a set of defining systems $A_{q}^{*}$ of $\langle a\rangle^{p}$, for $q=1, \cdots, p-1$, so that

(i) $a_{1}(n)=a(n)$,

(ii) $a_{q}(n)=a^{\prime}(n)$ for $n \leqq q$, and thus $a_{p-1}(n)=a^{\prime}(n)$ for $n=1, \cdots, p-1$,

(iii) the related cocycles of $A_{q}^{*}$ and $A_{q+1}^{*}$ are cohomologous. 
Assume inductively on $q$ that $\left(a_{q}(n)\right)$ is defined. Then $\delta a_{q}(q+1)=\delta a^{\prime}(q+1)$ so the two differ by a cocycle. Thus, by Lemma 16 , there is a defining system $A_{q+1}^{*}$ satisfying (i), (ii), (iii). Q.E.D.

By the preceding results, we may interpret the operation

$$
\langle\rangle^{p}: H^{2 m+1}\left(X ; Z_{p}\right) \rightarrow H^{2 m p+2}\left(X ; Z_{p}\right)
$$

as a primary operation of type $\left(Z_{p}, 2 m+1 ; Z_{p}, 2 m p+2\right)$. Also, by obvious scalar properties, if $g \in Z_{p},\langle g u\rangle^{p}=g^{p}\langle u\rangle^{p}=g\langle u\rangle^{p}$.

Now let $\mathrm{l}$ be the fundamental class of $H^{2 m+1}\left(Z_{p}, 2 m+1 ; Z_{p}\right)$. It is well known that $H^{*}\left(Z_{p}, 2 m+1 ; Z_{p}\right)$ is the tensor product of polynomial rings and exterior algebras each generated by $P^{I}(\mathfrak{l})$ for admissible sequences $I$ [1]. Therefore $\langle\mathfrak{l}\rangle^{p}=\Sigma c_{i} P^{I_{(i, 1)}(1) \cdots P^{I_{(i, j i)}}} \quad$ (l) with $j_{i} \geqq 1$ and $c_{i} \neq 0$ for all $i$.

Let $g$ be a primitive generator for the multiplicative group of $Z_{p}$. Then

$$
0=\langle g \mathfrak{l}\rangle^{p}-g\langle\mathfrak{l}\rangle^{p}=\Sigma c_{i}\left(g^{j_{l}}-g\right) P^{I_{(i, 1)}}(\mathfrak{l}) \cdots P^{I_{(i, j l}}(\mathfrak{i}) .
$$

This only happens if the coefficients vanish, i.e. $g^{j_{i}}-g=0$ in $Z_{p}$. Since $g$ is primitive, this is true if and only if $j_{i} \equiv 1(\bmod p-1)$ for each $i$. Since $\operatorname{dim} \imath^{p}>2 m p+2$, no (nontrivial) $p$-fold product can exist in $H^{2 m p+2}\left(Z_{p}, 2 m+1 ; Z_{p}\right)$. Therefore $j_{i}=1$ for all $i$; i.e. $\langle\mathrm{l}\rangle^{p}=\Sigma c_{i} P^{I_{i}}(\mathrm{l})$.

Now if $\sigma: H^{*}\left(Z_{p}, 2 m+1 ; Z_{p}\right) \rightarrow H^{*}\left(Z_{p}, 2 m ; Z_{p}\right)$ is the loop suspension, then by Theorem 5, $0=\sigma\langle\mathfrak{\imath}\rangle^{p}=\sum c_{i} P^{I_{i}}(\sigma \mathfrak{\imath})$ and thus $P^{I_{i}}(\sigma \mathfrak{\imath})=0$ for each $i$. The only Steenrod operation $P^{I}$ of degree $2 m p+2-2 m-1$ with this property is easily seen to be $\beta P^{m}$ (see [1]). We sum up these results in the following lemma for Theorem 14.

LEMMA 18. For some constant $c \in Z_{p},\langle u\rangle^{p}=c \beta P^{m} u$ for all $u \in H^{2 m+1}\left(X ; Z_{p}\right)$.

The next theorem will completely characterize the operation $\langle u\rangle^{p^{k}}$ for $k \geqq 1$ where $u$ is a one dimensional class $\bmod p$.

THEOREM 19. Let $\imath \in H^{1}\left(Z_{p^{k}}, 1 ; Z_{p}\right)$ be the $\bmod p$ reduction of the fundamental class $\mathfrak{1}_{k}$ of $H^{1}\left(Z_{p^{k}}, 1 ; Z_{p^{k}}\right)$. Then $\langle\imath\rangle^{p^{k}}$ is defined as the single class $-\beta_{k} \mathbf{l}_{k} \in H^{2}\left(Z_{p^{k}}, 1 ; Z_{p}\right)$, where $\beta_{k}$ is the Bockstein coboundary operator associated with the exact sequence of coefficient groups.

$$
0 \rightarrow Z_{p} \rightarrow Z_{p^{k+1}} \rightarrow Z_{p^{k}} \rightarrow 0 .
$$

Proof. Let $C^{*}\left(=C^{*}\left(Z_{p^{k}} ; Z_{p}\right)\right)$ be the cochain complex of the standard resolution of $Z_{p^{k}}$ with coefficients in $Z_{p}$. Then $C^{*}$ is cochain equivalent to the complex of singular cochains of a $K\left(Z_{p^{k}}, 1\right)$ space [2]. We use only the following properties of this complex:

(a) The cochains $a \in C^{n}$ are set maps from the $n$-fold Cartesian product $\left(Z_{p^{k}}\right)^{n}$ to $Z_{p}$, with the sole condition that $a\left(x_{1}, \cdots, x_{n}\right)=0$ whenever $x_{i}=0$ for some $i=1, \cdots, n$. 
(b) If $a \in C^{1}$, then $\delta a \in C^{2}$ is the map defined by $(\delta a)(x, y)=a(x)+a(y)-a(x+y)$.

(c) If $a, b \in C^{1}$, then the product $a b \in C^{2}$ is defined by $(a b)(x, y)=a(x) b(y)$.

We shall now explicitly construct a defining system of 1-cochains $(a(n))$ for $\langle i\rangle^{p^{k}}$. Let $a$ be the mod $p$ reduction of the "identity" map, i.e. $a(x)$ is the mod $p$ reduction of $x$. Then $a$ represents $t$.

For each $n=1, \cdots, p^{k}-1$, define $a(n)$ by $a(1)=a$ and by specifying that

$$
a(n)(x)=\left(\begin{array}{l}
\hat{x} \\
n
\end{array}\right)(\bmod p)
$$

This binomial coefficient is to be interpreted as follows: Let $\hat{x}$ be the integer $0 \leqq \hat{x}<p^{k}$ which represents $x$. Then

$$
\left(\begin{array}{l}
\hat{x} \\
n
\end{array}\right)
$$

is an integer, defined to be zero if $\hat{x}<n$. $a(n)(x)$ will be the mod-p reduction of this integer.

To verify the coboundary formula, we use a classical formula about binomial coefficients:

$$
\left(\begin{array}{c}
i+j \\
n
\end{array}\right)=\sum_{r=0}^{n}\left(\begin{array}{l}
i \\
r
\end{array}\right)\left(\begin{array}{c}
j \\
n-r
\end{array}\right)
$$

(Both are the coefficient of $t^{n}$ in $(1+t)^{i+j}=(1+t)^{i}(1+t)^{j}$.)

It suffices to verify (3.3), i.e.

$$
\left(\begin{array}{l}
\hat{x} \\
n
\end{array}\right)+\left(\begin{array}{l}
\hat{y} \\
n
\end{array}\right)-\left(\begin{array}{c}
(x+y)^{\wedge} \\
n
\end{array}\right)=-\sum_{r=1}^{n-1}\left(\begin{array}{l}
\hat{x} \\
r
\end{array}\right)\left(\begin{array}{c}
\hat{y} \\
n-r
\end{array}\right) \quad(\bmod p) .
$$

Now $\hat{x}+\hat{y}=(x+y)^{\wedge}+\varepsilon p^{k}$ where $\varepsilon=\varepsilon(x, y)$ is 0 or 1 . So by (3.6), the following formula holds:

$$
\sum_{r=0}^{n}\left(\begin{array}{l}
\hat{x} \\
r
\end{array}\right)\left(\begin{array}{c}
\hat{y} \\
n-r
\end{array}\right)=\sum_{r=0}^{n}\left(\begin{array}{c}
(x+y)^{\wedge} \\
r
\end{array}\right)\left(\begin{array}{c}
\varepsilon p^{k} \\
n-r
\end{array}\right) .
$$

Since $n<p^{k}$, the right hand side of (3.7) is

$$
\left(\begin{array}{c}
(x+y)^{\wedge} \\
n
\end{array}\right) \bmod p .
$$

Thus $(a(n))$ is defining system for $\langle\mathfrak{l}\rangle^{p}$. Furthermore

$$
c\left(A^{*}\right)(x, y)=-\sum_{r=0}^{p^{k}}\left(\begin{array}{l}
\hat{x} \\
r
\end{array}\right)\left(\begin{array}{c}
\hat{y} \\
p^{k}-r
\end{array}\right)=-\varepsilon(x, y) .
$$

To compute $\beta_{k} \mathfrak{l}_{k}$, take a fundamental cocycle $a^{\prime} \in C^{1}\left(Z_{p^{k}} ; Z_{p^{k}}\right)$ and pull it back to $a^{\prime \prime} \in C^{1}\left(Z_{p^{k}} ; Z_{p^{k+1}}\right)$. Then $\left(1 / p^{k}\right) \delta a^{\prime \prime}$ is a cocycle in $C^{2}\left(Z_{p^{k}} ; Z_{p}\right)$ which represents 
$\beta_{k} \mathfrak{l}_{k}$. We take $a^{\prime}$ to be defined by $a^{\prime}(x)=x$ and $a^{\prime \prime}(x)=\hat{x}$ in $Z_{p^{k+1}}$, i.e. $\hat{x}\left(\bmod p^{k+1}\right)$. Then

$$
\left(\delta a^{\prime \prime}\right)(x, y)=\hat{x}+\hat{y}-(x+y) \wedge\left(\bmod p^{k+1}\right)=\varepsilon(x, y) p^{k} .
$$

We need a few technical lemmas in order to complete the proof of Theorem 14. For a space $X$, coefficient ring $R$ and integer $k>2$, let $B_{k}$ denote the following condition:

$B_{k}$ - Whenever $v_{1}, \cdots, v_{r}$, for $r<k$, are odd dimensional classes of $H^{*}(X ; R)$ such that $\left\langle v_{1}, \cdots, v_{r}\right\rangle$ is defined, then $\left\langle v_{1}, \cdots, v_{r}\right\rangle$ consists solely of the zero element. It is clear that $B_{k}$ implies $B_{k-1}$. Also, if $v$ and $w$ are odd dimensional classes, then $B_{k}$ implies that $v w=0$.

Lemma 20. Assume that condition $B_{k}$ holds. If $u_{1}, \cdots, u_{k}$ are odd dimensional classes of $H^{*}(X ; R)$ such that $\left\langle u_{1}, \cdots, u_{k}\right\rangle$ is defined, then $\left\langle u_{1}, \cdots, u_{k}\right\rangle$ has no indeterminacy, i.e. it consists of a single class.

Proof. Let $a_{1}, \cdots, a_{k}$ be cocycle representatives of the classes, $u_{1} \cdots, u_{k}$ and let $A=(a(i, j))$ and $A^{\prime}=\left(a^{\prime}(i, j)\right)$ be two defining systems for $\left\langle a_{1}, \cdots, a_{k}\right\rangle$. We need to show that their related cocycles are cohomologous. First, note that $\operatorname{dim} a(i, j)$ is odd for all $i, j$.

To prove the lemma, it suffices to construct defining systems $A_{q}$ for $q=1, \cdots$, $k-1$ of $\left\langle a_{1}, \cdots, a_{k}\right\rangle$ so that

(i) $A_{1}=A$,

(ii) $a_{q}(i, j)=a^{\prime}(i, j)$ for $j-i \leqq q-1$ and thus $A_{k-1}=A^{\prime}$,

(iii) $c\left(A_{q}\right) \sim c\left(A_{q+1}\right)$ for $q=1, \cdots, k-2$.

We define these systems by induction on $q$. Assume that $A_{q}$ is defined. Then $b_{s}=a^{\prime}(s, q+s)-a_{q}(s, q+s)$ is a cocycle for each $s=1, \cdots, k-q$.

To construct $A_{q+1}$, it will suffice to construct defining systems $A_{q, s}$ of $\left\langle a_{1}, \cdots, a_{k}\right\rangle$ for $s=0,1, \cdots, k-q$ so that $a_{q, s}(i, j)=a_{q}(i, j)$ if $j-i \leqq q-1$,

$$
a_{q, s}(i, i+q)= \begin{cases}a_{q}(i, i+q) & \text { if } i>s, \\ a_{q}(i, i+q)+b_{i} & \text { if } i \leqq s,\end{cases}
$$

and $c\left(A_{q, s}\right) \sim c\left(A_{q, s+1}\right)$.Thus $A_{q}=A_{q, 0}$ and we may define $A_{q+1}$ to be $A_{q, k-q}$.

We define these systems by induction on $s$. Assume $A_{q, s}$ is defined. We will construct cochains $b(i, j)$ for $1 \leqq i \leqq j \leqq k$ so that we may define

$$
a_{q, s+1}(i, j)=a_{q, s}(i, j)+b(i, j) .
$$

If $i>s+1$ or $j<s+q+1$, then set $b(i, j)=0$. If $i \leqq s+1$ and $j \geqq s+q+1$, then we will construct the cochains $b(i, j)$ by induction on $j-i=q, \cdots, k-1$.

First set $b(s+1, s+q+1)=b_{s+1}$. Assume now that $b(i, j)$ is defined for all $(i, j)$ with $j-i<t$ so that 


$$
\delta b(i, j)=-\sum_{r=i}^{s} a_{q, s}(i, r) b(r+1, j)-\sum_{r=s+q+1}^{j-1} b(i, r) a_{q, s}(r+1, j) .
$$

Then the $b$ 's and the $a$ 's form defining systems for higher products with odd dimensional classes. In fact it is not hard to see that the right hand side of (3.9) is defined when $j=i+t$ and is a cocycle representative of the higher product $\left\langle a_{i}, \cdots, a_{s}, b_{s+1}, a_{s+q+2}, \cdots, a_{j}\right\rangle$. By condition $B_{k}$, this cocycle must cobound, i.e. there is a cochain $b(i, i+t)$ which satisfies (3.9).

It follows immediately from (3.8) and (3.9) that

$$
\delta b(i, j)=-\sum_{r=\imath}^{j-1} a_{q, s+1}(i, r) a_{q, s+1}(r+1, j)+\sum_{r=i}^{j-1} a_{q, s}(i, r) a_{q, s}(r+1, j)
$$

for all $i, j$ with $1 \leqq i \leqq j<k$. This shows both that $A_{q, s+1}$ is a defining system for $\left\langle a_{1}, \cdots, a_{k}\right\rangle$ and that the related cocycles of $A_{q, s}$ and $A_{q, s+1}$ are cohomologous. Q.E.D.

LEMMA 21. For some space $X$, ring $R$ and integer $k \geqq 2$, assume the following two conditions hold:

(3.10) If $u$ and $v$ are odd dimensional classes in $H^{*}(X ; R)$, then $u v=0$.

(3.11) If $u_{1}, \cdots, u_{r}$, for $r \leqq k$, are arbitrary odd dimensional classes of $H^{*}(X ; R)$, then $\left\langle u_{1}, \cdots, u_{r}\right\rangle$ is defined.

Then for arbitrary odd dimensional classes $u_{1}, \cdots, u_{r}, r \leqq k,\left\langle u_{1}, \cdots, u_{r}\right\rangle$ has no indeterminacy.

Proof. Clearly, we need only show that condition $B_{s}$ holds for $s=3,4, \cdots, k$. We shall prove this by induction on $s$. For $s=3$, it is trivial. Assume that condition $B_{s-1}$ holds. Since all $s$-fold products are defined, all $(s-1)$-fold products must contain the zero elements. By Lemma 20 , the $(s-1)$-fold products consist solely of the zero element. Thus condition $B_{s}$ holds. Q.E.D.

Proof of Theorem 14. Let $\mathrm{\imath}$ be the generator of $H^{1}\left(Z_{p}, 1 ; Z_{p}\right)$ and let $\beta \imath$ be the generator of $H^{2}\left(Z_{p}, 1 ; Z_{p}\right)$. Every odd dimensional class of $H^{*}\left(Z_{p}, 1 ; Z_{p}\right)$ can be written in the form $t v$ for some even dimensional class $v$. Since $\imath^{2}=0,(3.10)$ is satisfied. If $k \leqq p,\langle\imath\rangle^{k}$ is defined, and thus also the $k$-fold product $\langle\mathfrak{l}, \cdots, \mathfrak{l}\rangle$. Also if $v_{1}, \cdots, v_{k}$, are even dimensional classes, then by Corollary $7\left\langle\mathfrak{w} v_{1}, \cdots, \mathfrak{w} v_{k}\right\rangle$ is defined. Thus (3.11) is satisfied. By Lemma 21 all $p$-fold products in $H^{*}\left(Z_{p}, 1 ; Z_{p}\right)$ have no indeterminacy.

Thus, by Corollary 7 and Theorem 19,

$$
\begin{aligned}
\left\langle\mathfrak{l}(\beta \mathfrak{l})^{m}\right\rangle^{p} & =\left\langle\mathfrak{l}(\beta \mathfrak{l})^{m}, \cdots, \mathfrak{l}(\beta \mathfrak{l})^{m}\right\rangle \\
& =\langle\mathfrak{l}, \cdots, \mathfrak{l}\rangle(\beta \mathfrak{l})^{p m} \\
& =\langle\mathfrak{l}\rangle^{p}(\beta \mathfrak{l})^{p m} \\
& =-(\beta \mathfrak{l})^{p m+1} .
\end{aligned}
$$


By the Cartan formula,

$$
\begin{aligned}
\beta P^{m}\left(\mathfrak{l}(\beta \imath)^{m}\right) & =\beta\left(\mathfrak{l}(\beta \imath)^{p m}\right) \\
& =(\beta \imath)^{p m+1} .
\end{aligned}
$$

Since this element is nonzero in $H^{2 m p+2}\left(Z_{p}, 1 ; Z_{p}\right)$, the constant $c$ in Lemma 18 is - 1. Q.E.D.

\title{
BIBLIOGRAPHY
}

1. H. Cartan, Sur les groupes d'Eilenberg-MacLane, Proc. Nat. Acad. Sci. 40 (1954), 704-707.

2. S. Eilenberg and S. MacLane, Relations between homology and homotopy groups of a space, Ann. of Math. 46 (1945), 480-509.

3. G. Hirsch, Quelques propriétés des produits de Steenrod, C. R. Acad. Sci. Paris 241 (1955), 923-925.

4. D. Kraines, Higher products, Bull. Amer. Math. Soc. 72 (1966), 128-131.

5. W. S. Massey, Some higher order cohomology operations, Symposium International de Topologia Algebraica, pp. 145-154, La Universidad Nacional Autónama de México and UNESCO, Mexico City, 1958.

6. J. P. May, The cohomology of augmented algebras and generalized Massey products for DGA algebras (mimeographed), Yale University, New Haven, Conn., 1965.

7. E. Spanier, Algebraic topology, McGraw-Hill, New York, 1966.

8. — Higher order operations, Trans. Amer. Math. Soc. 109 (1963), 509-539.

9. J. D. Stasheff, Homotopy associativity of H-spaces, Trans. Amer. Math. Soc. 108 (1963), 293-312.

10. N. E. Steenrod, Products of cocycles and extensions of mappings, Ann. of Math. 48 (1947), 290-316.

11. H. Uehara and W. S. Massey, The Jacobi identity for Whitehead products, Algebraic geometry and topology, A symposium in honor of S. Lefschetz, Princeton Univ. Press, Princeton, N. J., 1957.

\author{
UNIVERSITY OF CALIFORNIA, \\ Berkeley, CALIFORNIA \\ Massachusetts Institute of Technology, \\ Cambridge, MassachusetTs
}

